\title{
Electrical impedance assays of blood cells
}

\author{
Eugen Gheorghiu* \\ International Centre of Biodynamics, Intrarea Portocalelor, 1B, 060101 Bucharest, Romania.
}

\begin{abstract}
In this review, the capability of electrical impedance spectroscopy analysis of blood cells, especially for red blood cells is presented, highlighting its large area of related biomedical relevance. The method is briefly introduced and basic theoretical aspects are discussed by considering both phenomenological (e.g. equivalent circuit) and microscopic approaches. The latter include a comparative analysis of the relevance of considering real shape (consistent with microscopic observations) versus spheroidal approximations (prolate and oblate spheroids) with the same surface and volume concentration. We show that while ellipsoidal approximation is fairly good for randomly oriented cells, it is quite poor whenever oriented cells are measured. The voluminous literature on the electrical analysis of blood cells is reviewed to stress the most promising biomedical applications of the method either per se or in combination with complementary e.g. (micro) fluidic approaches.
\end{abstract}

Keywords: electrical impedance spectroscopy, equivalent circuit, microscopic approach, prolate and oblate spheroids, red blood cell aggregation

Abbreviations: electrical impedance spectroscopy (EIS), red blood cells (RBCs), white blood cells (WBCs), red blood cell aggregation (RBCa), sample under test (SUT), geometric factor of the SUT(GESUT), mean corpuscular volume (MCV), mean corpuscular hemoglobin $(\mathrm{MCH})$, mean corpuscular hemoglobin concentration (MCHC), cardiovascular diseases (CVDs), prothrombin time (PT), partial thromboplastin time (PTT), and thrombin clotting time (TCT), electric impedance microflow cytometry (EIMC), sickle cell disease (SCD), acquired immunodeficiency syndrome (AIDS).

\section{INTRODUCTION}

Blood is a complex heterogeneous functional fluid that transports physiological gases as well as nutrients and metabolites throughout the body. It is primarily composed of red blood cells (RBCs), normally accounting for about $45 \%$ of the total blood volume. The rest consists of plasma, and other components representing less than $1 \%$ of the blood volume (white blood cells, platelets, etc.). Clearly, quantitative assessment of blood properties is highly relevant in medicine and biology. For diagnostic purposes, a plethora of in- vestigation methods addressing haematological and biochemical parameters of blood as well as the quest for various disease biomarkers have been advanced. These methods involve molecular, optical, (micro) fluidics or electrically (including electrochemically) assays $^{[1,2]}$. Despite the wide range of available technologies, when it comes to the blood of wild animals it is still difficult to find an easy and affordable solution for multiple species. The optical microscope was the primary method for cell classification and counting in human beings and animals. However, microscopy is still highly dependent on skilled laboratory personnel ${ }^{[3]}$.

*Correspondence to: Eugen Gheorghiu, International Centre of Biodynamics, Intrarea Portocalelor, 1B, 060101 Bucharest, Romania. E-mail: egheorghiu@biodyn.ro. 
The aim of this review is to highlight and summarize currently available electrically based methods to analyze blood components in relation to relevant biomedical aspects, as well as to emphasize several related (theoretical) limitations.

\section{ELECTRICAL IMPEDANCE SPECTROS- COPY}

Electrical impedance spectroscopy (EIS) is a powerful analytical method devised to characterize the non-homogeneities within a sample under test (SUT). EIS is carried out by applying a sine-wave voltage signal with frequency $v, V(t, v)$ and measuring the induced current, $I(t, v)$. The electrical impedance is the complex parameter defined as the voltage versus current ratio, as shown in eq. 1 :

$$
\begin{aligned}
& V^{*}(T, v)=V_{0}(v) \cdot \exp (2 \pi v t) ; I^{*}(t, v)=I_{0}(v) \cdot \exp (I \cdot(2 \pi v t+\phi)) \\
& Z_{S U T}^{*}(v)=\frac{V^{*}(t, v)}{I^{*}(t, v)}=\frac{V_{0}(v)}{I_{0}(v)} \exp (-I \phi) \\
& Z_{S U T}^{*}(v)=\operatorname{Re}\left(Z_{S U T}^{*}(v)\right)+I \operatorname{Im}\left(Z_{S U T}^{*}(v)\right) ;\left|Z_{S U T}^{*}(v)\right|=\frac{V_{0}(v)}{I_{0}(v)}
\end{aligned}
$$

The complex dielectric (permittivity) parameter is related to the complex impedance via the geometric factor of the SUT, $\mathrm{GF}_{\mathrm{SUT}}$.

$$
\varepsilon_{S U T}^{*}(v)=\frac{G F_{S U T}}{2 \pi v \cdot Z_{S U T}^{*}(v)} ; \varepsilon_{S U T}^{*}(v)=\varepsilon_{S U T}(v)+\frac{\sigma_{S U T}}{I 2 \pi v \cdot \varepsilon_{0}}
$$

$\varepsilon_{S U T}$ denotes the real part of SUT permittivity, $\sigma_{S U T}$ stands for SUT conductivity and $\varepsilon_{0}$ represents vacuum permittivity.

Aiming for label free blood analysis, electrical impedance assays have been extensively approached since the end of the 19th century, when Stewart ${ }^{[4,5]}$ discovered that the electrical conductance of blood decreases with increasing the erythrocyte concentration. The pioneering studies of Höber, Fricke and Cole ${ }^{[6-11]}$ revealed the equivalent circuit in RBCs, consisting of $\mathrm{Rp}$ (plasma resistance) in parallel with a series circuit given by Ri (resistance of cell interior) and $\mathrm{Cm}$ (membrane capacitance), from which the experimentally related electrical parameters were derived (Fig. 1).

RBC count plays an important role in animal diagnosis. Automatic counters that produce more specific

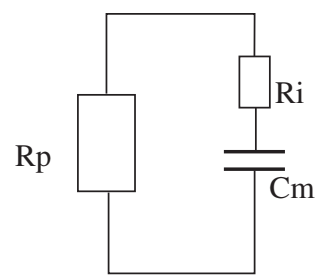

Fig. 1 Equivalent circuit of the red blood cells. It consists of Rp (plasma resistance) in parallel with a series circuit given by $\mathrm{Ri}$ (resistance of cell interior) and $\mathrm{Cm}$ (membrane capacitance). results and thus apply less subjective criteria were proposed. A revolutionary method for counting RBC based on the electrical impedance variation of the blood fluid and its suspended particles, when passing through a sensing aperture in a capillary was introduced by Coulter ${ }^{[12]}$.

Nurtured by both theoretical (via microscopic or phenomenological approaches) and experimental (including instrumentation) advancements, EIS has been extensively developed during the last century to become one of the first hand tools capable of noninvasively monitoring the electrical properties of cells and tissues ${ }^{[13-18]}$.

\section{THEORETICAL ASPECTS FOR DE- RIVING RED BLOOD CELL PARAM- ETERS FROM ELECTRICAL IMPED- ANCE MEASUREMENTS}

To derive quantitative data from electrical impedance measurements on cells and tissues, one has to consider either phenomenological approaches or microscopic models. While the phenomenological models are based on equivalent circuits comprising passive elements e.g. resistors and capacitors and/or mathematical expressions without a clear, intuitive physical representation ${ }^{[15,19]}$, the microscopic approaches provide potential and charge spatial distribution via analytical expressions derived using Maxwell equations and structural information from various investigation systems and approaches e.g. biological, optical microscopy, etc. Aiming to simplify analytical expressions, the rather complex shape of biological cells has been traditionally simplified to spheroidal if not to spherical ones. In the following we highlight the errors that may accompany spheroidal assessments instead of the actual shape of biological cells or tissues with emphasis on RBCs. While the detailed theory is presented in our previous papers ${ }^{[20-23]}$, here we discuss the shape effect on the dielectric (impedimetric) behavior of a suspension of RBCs. The whole range of RBC shapes (presenting axial symmetry) are simulated by considering the following expression:

$$
\left.\mathrm{R}[\theta]=\sqrt{-A \cdot \cos (2 \theta)+\sqrt{C^{2}-(A \sin (2 \theta))^{2}}} ; A=\frac{\left(d^{2}-t^{2}\right)}{8} ; C=\frac{\left(d^{2}+t^{2}\right)}{8}\right)
$$

Where $\theta$ denotes the polar angle, $d$ represents the $\mathrm{RBC}$ diameter (i.e. the distance between the lower and the upper points in the RBC contours, represented in Fig. 2) and t stands for the RBC thickness i.e. the distance between the points at the intersection of the $\mathrm{RBC}$ contours and the symmetry axes (the horizontal one) in Fig. 2.

Fig. 2 reveals the RBC shapes according to eq. 3 and the coordinates provided in Table 1. 
Table 1 Red blood cells parameters related to equation 3

\begin{tabular}{ccc}
\hline No. & Diameter $(\mu \mathrm{m})$ & Least thickness $(\mu \mathrm{m})$ \\
\hline 1 & 7 & 0.8 \\
2 & 6 & 1.0 \\
3 & 8 & 0.5 \\
\hline
\end{tabular}

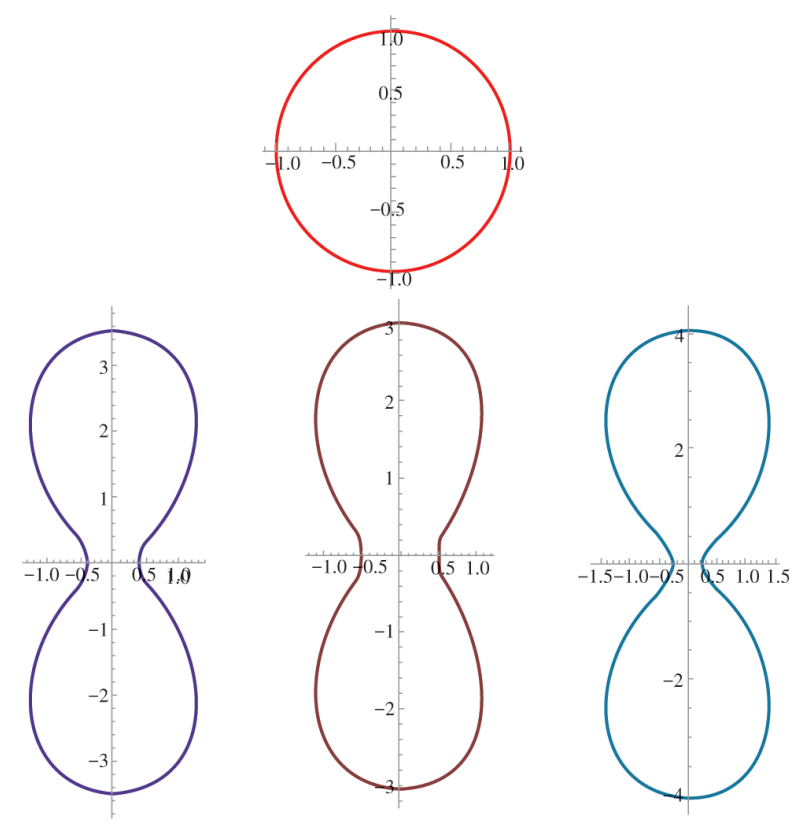

Fig. 2 The shapes considered for the red blood cells and a spherical one (one to three from left to right, with parameters in Table 1). The symmetry (rotation) axes is the horizontal one.

Considering a microscopic model ${ }^{[21]}$, one can derive the complex permittivity of a RBC suspension as given by:

$$
\varepsilon_{\text {sut }}=\varepsilon_{\text {out }}+p \cdot \frac{p \cdot \sum_{j} k_{j} \alpha_{j}}{1-p / 3 \cdot \sum_{j} k_{j} \alpha_{j}}
$$

Where $\varepsilon_{\text {out }}$ denotes the permittivity of the plasma, $p$ represents the volume ratio of the cells $(p=\mathrm{Vc} / \mathrm{Total}$ Volume) and $\alpha_{j}$ the polarizability corresponding to a specific orientation that has assigned the weight $k_{j}$ $(j=1 \div 3)$.

We have considered shelled particles (membrane thickness, $\mathrm{mt}=10^{-2} \mu \mathrm{m}$ ) with the following values for the electrical parameters: $\varepsilon^{*}{ }_{\text {in }}=70.1-$ I $0.2 /\left(2 \pi v \varepsilon_{0}\right)$, $\varepsilon_{\text {shell }}^{*}=12, \varepsilon_{0}^{*}=78-\mathrm{I} 0.377 /\left(2 \pi v \varepsilon_{0}\right)$ and $p=0.5 \mathrm{Vshape} /(4$ $\mathrm{Pi} / 3 \mathrm{Rsf}^{3}$ ); Rsf corresponds to the radius of the sphere with the same surface as the RBCs; the same surface and the same number of cells was assessed on all RBC shapes.

The dielectric behaviors of prolate and oblate spheroids (obtained by rotating an ellipse about its major axis, and minor axis respectively), having the same volume and surface as the actual red blood cell (with shapes described by eq.3) were computed and com- pared with the spectra of the real shapes. Whereas similar behavior (spectra) is derived when considering the suspension's randomly oriented cells $\left(k_{j}\right.$ $=1 / 3$ ), significant differences between simulations based on actual versus spheroidal shapes are obtained for oriented cells, especially when the electric field is perpendicular to the cell rotation axes (symmetry), as indicated in Fig. 3.

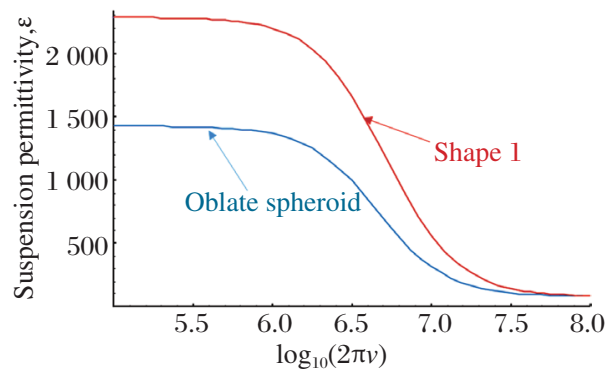

Fig. 3 The permittivity spectra for shape 1 (defined in Table 1) and the corresponding oblate spheroid when the electric field is perpendicular to the cell rotation axes. The electric field is perpendicular to the cell rotation/symmetry axes.

\section{USE OF ELECTRICAL IMPEDANCE MEASUREMENTS TO ASSESS BLOOD PROPERTIES AND RELATED BIO- MEDICAL/DIAGNOSIS RELEVANCE}

RBCs possess a unique capacity for undergoing cellular deformation to navigate across various human microcirculation vessels, enabling them to pass through capillaries that are smaller than their diameter and to carry out their role as gas carriers between blood and tissues. Since there is growing evidence that red blood cell deformability is impaired in some pathological conditions, measurement of RBC deformability has been the focus of numerous studies over the past decades ${ }^{[24]}$. Nevertheless, reports on healthy and pathological RBCs are currently limited and, in many cases, are not expressed in terms of well-defined cell membrane parameters such as elasticity and viscosity. Hence, it is often difficult to integrate these results into the basic understanding of RBC behavior, as well as into clinical applications ${ }^{[25]}$. Blood analysis is currently performed using various technologies that frequently include electrical impedance measurements and flow cytometry.

An impedance measurement system provides accurate and fast analysis of constituent components of a (liquid) sample. While electrical impedance still has a firm position in determining the overall number and size of cells, flow-cytometry techniques have proven 
their capability to differentiate white blood cells and identify abnormal cells ${ }^{[25]}$. Most automated blood cell counters assess blood parameters: hemoglobin content of RBCs, hematocrit, RBC count, mean corpuscular volume (MCV) of RBCs, mean corpuscular hemoglobin $(\mathrm{MCH})$, mean corpuscular hemoglobin concentration (MCHC), platelet count, mean platelet volume, and white blood cell(WBC) count. Quantitation of the RBCs, WBCs, and the platelets can be achieved by electrical impedance assay; following Coulter ${ }^{[12]}$, this approach relies on the conductance change accompanying cells passing through a small aperture ${ }^{[26]}$.

According to the World Health Organization, cardiovascular diseases (CVDs) account for about one third of total mortality all over the world. Patients with serious CVDs present microcirculatory disorders found to be strongly related with blood properties that are widely tested to diagnose the pathological or physiological disorders of $\mathrm{CVDs}^{[27]}$. Among the biophysical properties of blood, RBC aggregation is the major cause of the non-Newtonian flow properties of whole blood, and has been popularly utilized using electrical impedance spectroscopy to diagnose inflammatory diseases in clinic, since the level of aggregation rises enormously in association with diabetes, sepsis and myocardial ischemia ${ }^{[27-29]}$.

\section{RBC AGGREGATION (RBCA)}

$\mathrm{RBC}$ aggregation (in the form of rouleaux) can be altered during pathophysiological processes. Aggregation affects the flow properties of blood, especially at low shear rates, and therefore has the potential to influence blood flow in the circulatory system. There are several classes of RBC aggregation. Linear RBC aggregates are called rouleaux. The number of RBCs per rouleau can vary and branching into two rouleaux can occur ${ }^{[30]}$. Reversible rouleaux formation is caused by plasma macromolecules; however, several RBCs might become actively adhesive in the presence of a blood clot (e.g. caused by the activation of platelets). Clot formation can be life-saving in the case of wound healing, but also a major cause of death in the case of a thrombus induced stroke. Moreover, there are several pathological cases such as malaria or sickle cell diseases where red blood cells are known to form large aggregates that hinder the flow of blood ${ }^{[30]}$. The factors determining RBC aggregation are both cellular (e.g. RBC shape, glycocalyx, oxidant stress) and extracellular e.g. flow condition, $\mathrm{pH}$ value, osmotic pressure, concentration of plasma proteins (in acute phase reactions). Both in coagulation and aggregation, fibrinogen plays a crucial role; without any macromolecules, e.g. RBCs in a simple salt solution, no aggregation occurs ${ }^{[30,31]}$. Whereas the electrical properties of plasma and blood cells provide fundamental insights into the health status of patients, including the detection and evaluation of thrombus formation, the potential of EIS is not fully exploited today ${ }^{[32,33]}$.

Routine coagulation tests are set to provide rapid information on the general (relatively non-specific) nature of an abnormality and direct the clinician to a diagnosis by analyzing distinct coagulation factors. The parameters normally used to screen for haemostatic defects are prothrombin time (PT), partial thromboplastin time (PTT), and thrombin clotting time (TCT). PT is also widely used to monitor the treatment of patients receiving oral anticoagulant therapy. The data associated with the onset of coagulation provided by electrical impedance assays are comparable to those derived from absorbance measurements. The amplitude of the impedance change correlates well with the fibrinogen concentration of the plasma ${ }^{[34]}$. Quantitative assessment of blood coagulation is essential to predict the risk of hemorrhage and thrombosis during cardiac surgical procedures. Electrical impedance based devices integrating high throughput microfluidics were developed to assess blood coagulation time under temperature and hematocrit varia$\operatorname{tion}^{[35,36]}$.

Red blood cell aggregation is also a sensitive inflammation marker ${ }^{[37]}$. RBCA determination from erythrocyte sedimentation rate(ESR) is extensively used, but it proves unspecific unless corrected for hematocrit $(\mathrm{Ht})^{[38]}$. Moreover, whole blood viscosity measurement at low shear rate is also sensitive to RBCA but is cumbersome to apply. Electrical impedance of blood (sensitive to spatial RBC distribution) was used to determine RBCA in low shear conditions ${ }^{[39]}$. Shape and rigidity effects under different flow conditions were analyzed by studies combining both hemorheology (controlled shear flows) and electrical impedance assays ${ }^{[40]}$.

Whereas physiological properties of human erythrocytes in inflammation is an intensively studied topic, as recently highlighted ${ }^{[41]}$, the mechanism behind erythrocyte involvement in acute inflammation is not fully understood. As indicated in a couple of very comprehensive reviews ${ }^{[41,42]}$, sepsis induces profound changes in microcirculation with loss of capillary density, as well as by alterations in blood rheology resulting from decreased RBC and WBC deformability, RBC aggregation and coagulation disturbances. Moreover, sepsis relates to morphology changes, e.g. septic RBCs were found to be more spherical ${ }^{[43]}$ as opposed to the characteristic biconcave disc shape, as 
shown in Fig. 2 (second row).

\section{SHELF LIFE OF BLOOD}

Assessment of donor blood freshness (storage alterations) is another highly important biomedical issue that is currently supported by electrical impedance assays ${ }^{[4,45]}$. Monitoring the electrical impedance of blood during several weeks of storage at $4{ }^{\circ} \mathrm{C}^{[46]}$ revealed a decrease of both plasma resistance and the capacitance of cell membranes, while the resistance of the RBC interior fluid did not change significantly. These alterations are consistent with RBC lesions during storage and indicate that electrical impedance measurements are useful for monitoring RBC ageing and assessing the quality of stored RBCs. The membrane capacitance is a convenient parameter, allowing a quantitative measure of membrane state that can be otherwise studied with more complicated methods, such as electron microscopy and chemical analyses. Biomedical applications are supported by the differences in membrane capacitances of tumor tissues and of haemolyzed blood, which might be useful for evaluating the biocompatibility of blood related biomaterials ${ }^{[47]}$. Moreover, the shape of human red blood cells deteriorate progressively during hypothermic storage, with echinocytosis being the most prevalent pathway of this morphological lesion ${ }^{[48]}$. The change in shape of RBCs from normal discocytes progressively through various stages of echinocytosis to spherocytes produced a substantial decline in the ability of these cells to perfuse an artificial microvascular network $^{[49]}$. Echinocytosis induced by hypothermic storage could therefore be responsible for a similarly substantial impairment of deformability previously observed for stored $\mathrm{RBCs}^{[48]}$. Shape was proven to be essentially related to the decrease in the velocity difference between the cell and imposed flow, thus providing higher flow efficiency for RBCs. Higher membrane rigidity leads to a dramatic change in the slipper morphology, thus offering a potential diagnostic tool for cell pathologies ${ }^{[50]}$.

\section{HINTS ON BLOOD CELL RELATED DISEASES-RBC INFECTIONS (E.G. MALARIA), CHRONIC FATIGUE SYN- DROME, AND INHERITED BLOOD CELL DISORDER (ANEMIA)}

The electrical properties of biological cells reveal their healthy/pathological states. Electric impedance microflow cytometry (EIMC) can be used to characterize disease states of RBC. Such a platform comprises a microfluidic device for a label-free and non-invasive cell-counting assay through electric impedance sensing ${ }^{[51]}$. Invasion by Plasmodium falciparum induces physical and biochemical changes on host RBCs throughout a $48 \mathrm{~h}$ multi-stage life cycle within the $\mathrm{RBC}^{[52]}$. As such, it also induces progressive changes in the electrical properties of host cells. It was demonstrated that the EIMC system in combination with data analysis allows differentiation of $P$. falciparum infected RBCs from uninfected ones, as well as among different $P$. falciparum intra-erythrocytic asexual stages including the ring stage ${ }^{[52]}$.

Recently, a novel electrical impedance test for the diagnosis of a challenging disease i.e. myalgic encephalomyelitis/chronic fatigue syndrome based on disease related blood cell modification was proposed $^{[53]}$.

Sickle cell disease (SCD) is a common inherited blood cell disorder that affects hemoglobin. The disease state of a sickle red blood cell is closely related to the intracellular hemoglobin composition and concentration. A mutation in the $\beta$-globin gene changes the hydrophilic glutamic acid to a strongly hydrophobic valine amino acid, resulting in abnormal hemoglobin $\mathrm{S}(\mathrm{HbS})^{[54]}$. At low oxygen tension, HbS polymerizes and forms rigid fibers, giving rise to rigid $\mathrm{RBCs}$ with distorted cell membranes, known as cell sickling. These rigid sickled cells adversely affect blood circulation and oxygen transport efficiency, and have been associated with anemia severity and vaso-occlusive manifestations in various sickling syndromes ${ }^{[54]}$. The standard diagnosis of SCD is based on hemoglobin analysis, which typically requires a hemolysate using a hemoglobin analyzer. An electrically based microflow cytometry method, with oxygen control for the detection of sickle cells was recently proposed; its results indicate that electrical impedance signals are able to differentiate sickle cells from normal cells ${ }^{[54,55]}$.

\section{BLOOD CELL ANALYSIS FOR MONI- TORING DISEASE PROGRESSION}

CD4 ${ }^{+}$T-lymphocyte count is a widely used method for monitoring acquired immunodeficiency syndrome (AIDS) progression, staging, and response to drug therapy in human immunodeficiency virus(HIV) infected individuals in resource poor settings. According to WHO guidelines, a $\mathrm{CD} 4^{+} \mathrm{T}$-lymphocyte count of fewer than 200 cells/ $\mu \mathrm{L}$ in whole blood establishes the diagnosis of AIDS. Single cell microfluidic impedance cytometry has been used to identify cells at high speed, on the basis of their dielectric properties, however it cannot be used to identify subpopulations of cells (there is no electrical analogue to a fluorescent label). For changing the electrical properties of a 
target subset of cells, small antibody conjugated beads are mixed with cells to bind to the target population. This method was used to discriminate and quantitate antigenically defined $\mathrm{CD} 4^{+} \mathrm{T}$-lymphocyte subpopulation in human whole blood by an electrical impedance assay $^{[56]}$.

Recent advances in the mechanisms of platelet activation and potential applications of platelet activation biomarkers to diagnose and predict disease states were recently reviewed and discussed ${ }^{[57,58]}$. Accordingly, several markers of platelet activation have been identified to correlate with the presence of inflammation and atherosclerosis. Since these markers have relatively short detectability in circulating blood, plateletmonocyte aggregates have recently emerged as markers for platelet activation ${ }^{[59]}$.

\section{CONCLUDING REMARKS}

The electrical impedance (or related dielectric/permittivity) spectra of blood can provide relevant biomedical information. When using simple models (e.g. equivalent circuit, as in Fig. 1) one can derive plasma resistance as well as membrane capacitance that directly correlate with the erythrocyte sedimentation rate and can be also used as valuable quantitative tool to assess the quality of stored blood. More intricate (e.g. microscopic) approaches can reveal RBC shape as well as quantitate RBC aggregation (both rouleaux and clot formation) with high biomedical relevance.

Electrical impedance assays actually measure several RBC parameters, i.e. concentration, size, electrical properties to assess cell aging, in particular $\mathrm{Rp}$ (plasma resistance) and $\mathrm{Cm}$ (membrane capacitance), as represented in Fig. 1. As with aggregation and shape (when jointly used with a method able to control cell orientation versus the direction of the electric field), other parameters with biomedical relevance (e.g. deformability, membrane rigidity) require combined approaches with complementary methods involving (micro) fluidics including rheology assays, as highlighted within the cited Biomicrofluidics and Hemorheology reports.

Having in view the rather wide medical applicability of EIS data highlighted in the previous section, either per se or in combination with (micro) fluidics in lab-on-a-chip devices, EIS is expected to further evolve to become an ubiquitous effective, non-invasive tool to support rapid diagnosis in point of care units.

\section{References}

[1] Pradhan R, Mitra A, Das S. Impedimetric characterization of human blood using three-electrode based ECIS
devices[J]. J Electr Bioimpedance, 2012, 3: 12-9.

[2] Song Y, Huang YY, Liu X, et al. Point-of-care technologies for molecular diagnostics using a drop of blood[J]. Trends Biotechnol, 2014, 32(3): 132-9.

[3] Mauricio CRM, Schneider FK, Takahira RK, et al. Imagebased red blood cell counter for multiple species of wild and domestic animals[J]. Arq Bras Med Vet Zootec, 2017, 69: 75-84.

[4] Zhao TX. New applications of electrical impedance of human blood[J]. J Med Eng Technol, 1996, 20(3): 115-20.

[5] Stewart GN. Researches on the circulation time and on the influences which affect it[J]. J Physiol, 1897, 22: 15883.

[6] Höber R. Ein zweites verfahren die leitfaehigkeit im innern von zellen ze messem[J]. Arch Ges Physiol, 1912, 148: 189-221.

[7] Fricke H. A mathematical treatment of the electric conductivity and capacity of disperse systems[J]. Phys Rev, 1925, 26: 678-81.

[8] Fricke H. The electric capacity of suspensions with special reference to blood[J]. J Gen Physiol, 1925, 9: 13752.

[9] Fricke H. The electric impedance of haemolysed suspensions of mammalian erythrocytes[J]. J Gen Physiol, 1934, 18: 821-36.

[10] Fricke H, Morse S. The electric resistance and capacity of blood for frequencies between 800 and 4.5 million cycles[J]. J Gen Physiol, 1925, 9: 153-67.

[11] Cole KS. Electric impedance of suspensions of spheres[J]. J Gen Physiol, 1928, 12: 29-36.

[12] Coulter WH. Means for counting particles suspended in a fluid[P]. Patent 2.656.508, 27 Aug. 1949, 20 Oct. 1953. Chicago, Ill: United States Patent Office, 1953.

[13] Schwan HP. Electrical properties of blood and its constituents-alternating current spectroscopy[J]. Blut, 1983, 46: 185-97.

[14] Wolf M, Gulich R, Lunkenheimer P, et al. Broadband dielectric spectroscopy on human blood[J]. Biochim Biophys Acta Gen Subj, 2011, 1810(8): 727-40.

[15] Vosika ZB, Lazovic GM, Misevic GN, et al. Fractional calculus model of electrical impedance applied to human skin[J]. PLoS One, 2013, 8(4): e59483.

[16] David S, Polonschii C, Gheorghiu M, et al. Assessment of pathogenic bacteria using periodic actuation[J]. $L a b$ Chip, 2013, 13:3192-8.

[17] Gheorghiu E. Characterizing cellular systems by means of dielectric spectroscopy[J]. Bioelectromagnetics, 1996, 17(6): 475-82.

[18] Gheorghiu E, Asami K. Monitoring cell cycle by impedance spectroscopy: experimental and theoretical aspects[J]. Bioelectrochem Bioenerg, 1998, 45: 139-43.

[19] Gheorghiu M, Gersing E, Gheorghiu E. Quantitative analysis of impedance spectra of organs during ischemia[J]. Ann N Y Acad Sci, 1999, 873: 65-71.

[20] Vrinceanu D, Gheorghiu E. Shape effects on the dielectric behaviour of arbitrarily shaped particles with particular reference to biological cells[J]. Bioelectrochem 
Bioenerg, 1996, 40: 167-70.

[21] Gheorghiu E. On the limits of ellipsoidal models when analyzing dielectric behavior of living cells emphasis on red blood cells[J]. Ann N Y Acad Sci, 1999, 873:262-8.

[22] Gheorghiu E, Balut C, Gheorghiu M, Dielectric behaviour of gap junction connected cells: a microscopic approach[J]. Phys Med Biol, 2002, 47: 341-8.

[23] Sandu T, Vrinceanu D, Gheorghiu E. Linear dielectric response of clustered living cells[J]. Phys Rev EStat Nonlin Soft Matter Phys, 2010, 81: 0219131.

[24] Huisjes R, Bogdanova A, van Solinge WW, et al. Squeezing for life-properties of red blood cell deformability[J]. Front Physiol, 2018, 9: 656.

[25] Tomaiuolo G. Biomechanical properties of red blood cells in health and disease towards microfluidics[J]. Biomicrofluidics, 2014, 8: 051501.

[26] Sullivan E. Hematology analyzer: From workhorse to thoroughbred[J]. Lab Med, 2006, 37: 273-8.

[27] Wen J, Wan N, Bao H, et al. Quantitative measurement and evaluation of red blood cell aggregation in normal blood based on a modified Hanai equation[J]. Sensors, 2019, 19(5): 1095.

[28] Lei KF, Chen KH, Tsui PH, et al. Real-time electrical impedimetric monitoring of blood coagulation process under temperature and hematocrit variations conducted in a microfluidic chip[J]. PLoS One, 2013, 8(10): e76243.

[29] Üyüklü M. Measurement of impedance values of different erythrocyte suspensions[J]. Bezmialem Science, 2019, 7(3): 233-9.

[30] Wagner C, Steffen P, Svetina S. Aggregation of red blood cells: from rouleaux to clot formation[J]. Comptes Rendus Physique, 2013, 14: 459.

[31] Derganc J, Bozic B, Svetina S, et al. Equilibrium shapes of erythrocytes in rouleaux formation[J]. Biophys $J$, 2003, 84: 1486-92.

[32] Zhbanov A, Yang S. Electrochemical impedance spectroscopy of blood for sensitive detection of blood hematocrit, sedimentation and dielectric properties[J]. Anal Methods, 2017, 9: 3302-13.

[33] Li J, Wan N, Wen J, et al. Quantitative detection and evaluation of thrombus formation based on electrical impedance spectroscopy[J]. Biosens Bioelectron, 2019, 141: 111437.

[34] Spence N. Electrical impedance measurement as an endpoint detection method for routine coagulation tests[J]. Br J Biomed Sci, 2002, 59(4): 223-7.

[35] Lei KF, Chen KH, Tsui PH, et al. Real-time electrical impedimetric monitoring of blood coagulation process under temperature and hematocrit variations conducted in a microfluidic chip[J]. PLoS One, 2013, 8, e76243.

[36] Kang YJ. Microfluidic-based measurement method of red blood cell aggregation under hematocrit variations[J]. Sensors, 2017, 17: 2037.

[37] Flormann D. Physical characterization of red blood cell aggregation[D]. Homburg: Saarland University, 2017
[38] Pop GAM, Hop WJ. Blood electrical impedance closely matches whole blood viscosity as parameter of hemorheology and inflammation[J]. Appl Rheol, 2003, 13: 305-12.

[39] Baskurt OK, Baskurt OK, Meiselman HJ. Time course of electrical impedance during red blood cell aggregation in a glass tube: Comparison with light transmittance[J]. IEEE Trans Biomed Eng, 2010, 57: 969-78.

[40] Balan C, Balut C, Gheorghe L, et al. Experimental determination of blood permittivity and conductivity in simple shear flow[J]. Clin Hemorheol Microcirc, 2004, 30: 359.

[41] Saldanha C, Silva-Herdade AS. Physiological properties of erythrocytes in inflammation[J]. J Cell Biotechnol, 2017, 3: 15-20.

[42] Piagnerelli M, Boudjeltia KZ, Vanhaeverbeek M, et al. Red blood cell rheology in sepsis[J]. Intensive Care Med, 2003, 29: 1052-61.

[43] Bateman RM, Sharpe MD, Singer M, et al. The effect of sepsis on the erythrocyte[J]. Int J Mol Sci, 2017, 18(9): 1932-55.

[44] Simsek FG, Ulgen Y. Electrical impedance of human blood with and without anticoagulants in the $\beta$ dispersion region[J]. Eng Med Biol Soc, 2012, 2012: 3262-4.

[45] Ulgen Y, Sezdi M. Physiological quality assessment of stored whole blood by means of electrical measurements[J]. Med Bio Eng Comput, 2007, 45: 65360.

[46] Zhao TX, Shanwell A. Electrical impedance alterations of red blood cells during storage[J]. Vox Sang, 1994, 66: 258-63.

[47] Zhao TX, Jacobson B, Ribbe T. Triple frequency method for measuring blood impedance[J]. Physiol Meas, 1993, 14: $145-56$.

[48] Piety NZ, Reinhart WH, Pourreau PH, et al. Shape matters: the effect of red blood cell shape on perfusion of an artificial microvascular network[J]. Transfusion, 2016, 56(4): 844-51.

[49] Sosa JM, Nielsen ND, Vignes SM, et al. The relationship between red blood cell deformability metrics and perfusion of an artificial microvascular network[J]. Clin Hemorheol Microcirc, 2013, 57: 275-89.

[50] Kaoui B, Biros G, Misbah C. Why do red blood cells have asymmetric shapes even in a symmetric flow?[J]. Phys Rev Let, 2009, 103(18): 188101.

[51] Du E, Ha S, Diez-Silva M, et al. Electric impedance microflow cytometry for characterization of cell disease states[J]. Lab Chip, 2013, 13(19): 3903-9.

[52] Ribaut C, Reybier K, Reynes O, et al. Electrochemical impedance spectroscopy to study physiological changes affecting the red blood cell after invasion by malaria parasites[J]. Biosens Bioelectron, 2009, 24: 2721-5.

[53] Esfandyarpour R, Kashi A, Nemat-Gorgani M, et al. A nano electronics-blood-based diagnostic biomarker for myalgic encephalomyelitis /chronic fatigue syndrome (ME/CFS)[J]. Proc Natl Acad Sci USA, 2019, 116: 10250-7.

[54] Liu J, Qiang Y, Alvarez O, et al. Electrical impedance 
microflow cytometry with oxygen control for detection of sickle cells[J]. Sens Actuators B, 2018, 255: 2392-8.

[55] Liu J, Qiang Y, Alvarez O, et al. Electrical impedance characterization of erythrocyte response to cyclic hypoxia in sickle cell disease[J]. ACS Sensors, 2019, 4(7): 1783-90.

[56] Holmes D, Morgan H. Single cell impedance cytometry for identification and counting of CD4 T-cells in human blood using impedance labels[J]. Anal Chem, 2010, 82: 1455-61.

[57] Yun SH, Sim EH, Goh RY, et al. Platelet activation: The mechanisms and potential biomarkers[J]. Biomed Res Int, 2016, 2016: 9060143.

[58] Ghoshal K, Bhattacharyya M. Overview of platelet physiology: its hemostatic and nonhemostatic role in disease pathogenesis[J]. Sci World J, 2014, 2014: 781857.

[59] Mitsui C, Kajiwara K, Hayashi H, et al. Platelet activation markers overexpressed specifically in patients with aspirin-exacerbated respiratory disease[J]. J Allergy Clin Immunol, 2016, 137(2): 400-11.

Received 29 August 2019, Revised 11 November 2019, Accepted 17 January 2020 